\title{
Metric Entropy of Convex Hulls
}

\author{
Fuchang Gao \\ University of Idaho
}

\begin{abstract}
Let $T$ be a precompact subset of a Hilbert space. The metric entropy of the convex hull of $T$ is estimated in terms of the metric entropy of $T$, when the latter is of order $\alpha=2$. The estimate is best possible. Thus, it answers a question left open in $[\mathrm{LL}]$ and $[\mathrm{CKP}]$.
\end{abstract}

\subsection{Introduction}

Let $H$ be a separable Hilbert space and let $T$ be a precompact subset. Define the covering number

$$
N(T, \varepsilon):=\inf \left\{n: \exists t_{1}, t_{2}, \ldots, t_{n} \in T \text {, s.t. } T \subset \bigcup_{k=1}^{n} B\left(t_{k}, \varepsilon\right)\right\}
$$

where $B(x, \varepsilon)$ is the open $\varepsilon$-ball centered at $x \in H$. The set

$$
N_{\varepsilon}(T):=\left\{t_{1}, t_{2}, \ldots, t_{n}\right\}
$$

is called an $\varepsilon$-net of $T$. The quantity $\log N(T, \varepsilon)$ plays an important role in the theory of empirical processes (cf.[D]). It is called the metric entropy of $T$.

Let $\operatorname{cov}(\mathrm{T})$ denote the convex hull of $T$. It is natural to ask for good estimates of $\log N(\operatorname{cov}(T), \varepsilon)$ in terms of $\log N(T, \varepsilon)$. It is known (cf. [C]) 
that if $\log N(T, \varepsilon)<c \cdot \varepsilon^{-\alpha}$ for some $\alpha>0$, then

$$
\begin{aligned}
& \log N(\operatorname{cov}(T), \varepsilon) \leq c \cdot \varepsilon^{-2}\left(\log \varepsilon^{-1}\right)^{1-2 / \alpha}, \quad 0<\alpha<2, \\
& \log N(\operatorname{cov}(T), \varepsilon) \leq c \cdot \varepsilon^{-\alpha}, \quad \alpha>2 \text {, }
\end{aligned}
$$

and those are best possible. As we can see from the above that the situation is completely different for $\alpha<2$ and $\alpha>2$. The case $\alpha=2$ was open. In [LL], Li and Linde studied the metric entropy of $\operatorname{cov}(T)$ via certain quantities originated in the theory of majorizing measures. Among others, they obtained some finer estimates of $\log N(\operatorname{cov}(T), \varepsilon)$, which lead to some important partial results for $\alpha=2$. For example, the upper bounds for the entropy of $\operatorname{cov}(T), T=\left\{t_{1}, t_{2}, \ldots\right\},\left\|t_{i}\right\| \leq a_{i}$, by functions of the $a_{i}$ 's only. Their results are optimal for the slowly decreasing sequence $\left(a_{i}\right)$. However, in general, the estimate of the metric entropy of $\operatorname{cov}(T)$ for the case $\alpha=2$ was left open.

In this paper, we give the best possible estimate for the case $\alpha=2$. More precisely, we prove the following

Theorem 1 Let $H$ be a separable Hilbert space and let $T$ be a precompact subset of $H$.

(i) Suppose $\log N(T, \varepsilon)<\varepsilon^{-2}$, then for some $c>0$,

$$
\log N(\operatorname{cov}(T), \varepsilon) \leq c \cdot \varepsilon^{-2}\left(\log \varepsilon^{-1}\right)^{2} ;
$$

(ii) There exists a set $T$, and a constant $c>0$, such that

$$
\sup _{\varepsilon>0} \varepsilon^{2} \log N(T, \varepsilon) \leq 8,
$$


and for all $\varepsilon<c$,

$$
\log N(\operatorname{cov}(T), \varepsilon) \geq c \varepsilon^{-2}\left(\log \left(\varepsilon^{-1}\right)\right)^{2} .
$$

\subsection{Proof of (i)}

Without loss of generality, we assume the diameter of $T$ is 1 . For $k \geq 1$, let $N_{k}$ be a $2^{-k}$-net of $T$ with minimal cardinality. Denote $D_{1}=N_{1} \cup\{0\}$ and

$$
D_{n}=\left\{z \in N_{n}-N_{n-1}:\|z\| \leq 2^{-n+1}\right\} \cup\{0\}
$$

for $n>1$. Then

$$
T \subset D_{1}+D_{2}+\cdots+D_{n}+\cdots
$$

where "+" means the Minkowsky sum. By the assumption of (i), $D_{n}$ consists of no more than $e^{c 2^{2 n}}$ vectors for some constant $c>0$. Denote $C_{n}=\operatorname{cov}\left(D_{n}\right)$ and $E_{n}=C_{1}+C_{2}+\cdots+C_{n}$, then we have

$$
\operatorname{cov}(T) \subset C_{1}+C_{2}+\cdots+C_{n}+\cdots=E_{n}+C_{n+1}+\cdots
$$

For any $0<\varepsilon<1 / 4$, suppose $2^{-n+2} \leq \varepsilon<2^{-n+3}$. Because $C_{n+1}+$ $C_{n+2}+\cdots$ has diameter at most $2^{-n+1}$, we have

$$
\log N(\operatorname{cov}(T), \varepsilon) \leq \log N\left(E_{n}, 2^{-n+1}\right) .
$$

To estimate the right side above, we need the following lemma, whose proof is standard.

Lemma 1 There exists a constant $c$, such that for any $\lambda>0$,

$$
\log N\left(E_{n}, \lambda\right) \leq c n^{2} \cdot \lambda^{-2} .
$$


Proof: $\quad$ For each $k \leq n$, suppose $D_{k}=\left\{x_{1}, x_{2}, \ldots, x_{d_{k}}\right\}$, where $d_{k}$ is the cardinality of $D_{k}$. Thus, $d_{k} \leq e^{c 2^{2 k}}$. For each $z_{k} \in C_{k}, z_{k}$ can be expressed as

$$
z_{k}=\sum_{i=1}^{d_{k}} a_{i} x_{i}, a_{i} \geq 0, \quad \sum_{i=1}^{d_{k}} a_{i} \leq 1 .
$$

Define random vector $Z_{k}$, so that

$$
\operatorname{Pr}\left(Z_{k}=x_{i}\right)=a_{i}, 1 \leq i \leq d_{k}, \text { and } \operatorname{Pr}\left(Z_{k}=0\right)=1-\sum_{i=1}^{d_{k}} a_{i} .
$$

Let $Z_{k, 1}, Z_{k, 2}, \ldots, Z_{k, m_{k}}$ and $Z_{k, 1}^{\prime}, Z_{k, 2}^{\prime}, \ldots, Z_{k, m_{k}}^{\prime}$ be independent copies of $Z_{k}$. Then

$$
E \frac{1}{m_{k}} \sum_{i=1}^{m_{k}} Z_{k, i}=z_{k}
$$

Thus, by convexity and symmetrization, we have

$$
\begin{aligned}
E\left\|\sum_{k=1}^{n} z_{k}-\sum_{k=1}^{n} \frac{1}{m_{k}} \sum_{i=1}^{m_{k}} Z_{k, i}\right\| & =E\left\|E^{\prime} \sum_{k=1}^{n} \frac{1}{m_{k}} \sum_{i=1}^{m_{k}} Z_{k, i}^{\prime}-\sum_{k=1}^{n} \frac{1}{m_{k}} \sum_{i=1}^{m_{k}} Z_{k, i}\right\| \\
& \leq E E^{\prime}\left\|\sum_{k=1}^{n} \frac{1}{m_{k}} \sum_{i=1}^{m_{k}}\left(Z_{k, i}^{\prime}-Z_{k, i}\right)\right\| \\
& =E E^{\prime}\left\|\sum_{k=1}^{n} \frac{1}{m_{k}} \sum_{i=1}^{m_{k}}\left(Z_{k, i}^{\prime}-Z_{k, i}\right) r_{k, i}(t)\right\| \\
& \leq 2 E\left\|\sum_{k=1}^{n} \frac{1}{m_{k}} \sum_{i=1}^{m_{k}} Z_{k, i} r_{k, i}(t)\right\|
\end{aligned}
$$

where $\left(r_{k, i}(t)\right), 1 \leq k \leq n, 1 \leq i \leq m_{k}$, is a Rademacher sequence. Integrating with respect to $t$ over $[0,1]$, and using Fubini, we obtain

$$
\begin{aligned}
E\left\|\sum_{k=1}^{n} z_{k}-\sum_{k=1}^{n} \frac{1}{m_{k}} \sum_{i=1}^{m_{k}} Z_{k, i}\right\| & \leq 2 E\left(\sum_{k=1}^{n} \frac{1}{m_{k}^{2}} \sum_{i=1}^{m_{k}}\left\|Z_{k, i}\right\|^{2}\right)^{1 / 2} \\
& \leq 2\left(\sum_{k=1}^{n} \frac{1}{m_{k}} 2^{-2 k+2}\right)^{1 / 2}=\lambda
\end{aligned}
$$


taking $m_{k}=4 n 2^{-2 k+2} \lambda^{-2}$. This in particular implies that for some realization,

$$
\left\|\sum_{k=1}^{n} z_{k}-\sum_{k=1}^{n} \frac{1}{m_{k}} \sum_{i=1}^{m_{k}} Z_{k, i}\right\| \leq \lambda .
$$

But, there is no more than

$$
\prod_{k=1}^{n}\left(d_{k}\right)^{m_{k}} \leq e^{c n^{2} \lambda^{-2}}
$$

possible realizations of $\sum_{k=1}^{n} \sum_{i=1}^{m_{k}} Z_{k, i} / m_{k}$. The lemma follows.

Applying Lemma 1 with $\lambda=2^{-n+1}$, and keeping in mind that $2^{-n+2} \leq$ $\varepsilon<2^{-n+3}$, we obtain

$$
\begin{aligned}
\log N(\operatorname{cov}(T), \varepsilon) & \leq \log N\left(E_{n}, 2^{-n+1}\right) \\
& \leq c \cdot n^{2} 2^{-2 n+2} \\
& =c^{\prime} \varepsilon^{-2}\left(\log \varepsilon^{-1}\right)^{2}
\end{aligned}
$$

Remark 1 Both Li and Linde pointed out to me that the result (i) can be derived from a result in $[C K P]$. We include the proof because the current proof seems more transparent, and holds for any Banach space of type 2. Also, it is more convenient to the readers.

\subsection{Proof of (ii)}

Let $\left(\mathbf{e}_{k}\right)$ be a standard basis of $H$. For each integer $k \geq 1$, we define

$$
D_{k}=\left\{2^{-k} \mathbf{e}_{i}: e^{2^{2 k-2}} \leq i \leq e^{2^{2 k}}\right\} \cup\{0\}
$$


and $T=D_{1}+D_{2}+\cdots+D_{k}+\cdots$. For any $0<\varepsilon<1$, suppose $2^{-n} \leq \varepsilon<$ $2^{-n+1}$. Define $S_{n}=D_{1}+D_{2}+\cdots+D_{n}$. Because $S_{n}$ is an $2^{-n}$-net of $T$, and $S_{n}$ has cardinality no more than

$$
\prod_{k \leq n} e^{2^{2 k}} \leq e^{2^{2 n+1}}
$$

we have $\log N(T, \varepsilon)<2^{2 n+1}$. Thus

$$
\varepsilon^{2} \log N(T, \varepsilon)<2^{-2 n+2} \cdot 2^{2 n+1}=8 .
$$

To obtain a lower bound for $\log N(\operatorname{cov}(T), \varepsilon)$, we need the following lemma.

Lemma 2 There exists $c>0$, such that for $e^{-2^{2 k-3}}<\delta<c \cdot 2^{-k}$,

$$
\log N\left(\operatorname{cov}\left(D_{k}\right), \delta\right)>c \cdot \delta^{-2}
$$

Proof: Denote $I_{k}=\left\{i: e^{2^{2 k-2}} \leq i<e^{2^{2 k}}\right\}$, and let $\left|I_{k}\right|$ be the cardinality of $I_{k}$. Consider the set

$$
A=\left\{\sum_{i \in I_{k}} a_{i} \varepsilon \mathbf{e}_{i}: a_{i} \text { is non-negative integer, } \sum_{i \in I_{k}} a_{i} \leq 2^{-k} / \varepsilon\right\} .
$$

Let $m$ be the largest integer, such that $m \leq 2^{-k} / \varepsilon$. Then $A$ has cardinality no less than $\left|I_{k}\right|^{m} / m !>\left|I_{k}\right|^{m / 2}$. For each $t \in A$, and $2 \leq l<m$, consider

$$
B(t, l)=\left\{s \in A:\|t-s\|_{1} \leq l \varepsilon\right\} .
$$

$B(t, l)$ contains no more than $2^{l}\left|I_{k}\right|^{l} \leq\left|I_{k}\right|^{2 l}$ elements. Thus $A$ contains a subset $U$ of cardinality more than $\left(\left|I_{k}\right|^{m / 2}\right) \div\left(\left|I_{k}\right|^{2 l}\right)$, whose mutual $l_{1^{-}}$ 
distance between any two elements is at least $l \varepsilon$. Thus, the mutual $l_{2^{-}}$ distance is at least $\sqrt{l} \varepsilon$. Let $l \approx m / 6$. Because $A \subset \operatorname{cov}\left(D_{k}\right)$, we have

$$
\begin{aligned}
\log N\left(\operatorname{cov}\left(D_{k}\right), \sqrt{l} \varepsilon\right) & \leq \log N\left(\operatorname{co}\left(D_{k}\right), \sqrt{m / 6} \cdot \varepsilon\right) \\
& \geq \log \left(\left|I_{k}\right|^{m / 2} /\left|I_{k}\right|^{m / 3}\right) \\
& \geq \frac{m}{6} \log \left|I_{k}\right|
\end{aligned}
$$

which implies that $\log N\left(\operatorname{cov}\left(D_{k}\right), \delta\right) \geq c \cdot \delta^{-2}$ for some $c>0$ and $e^{-2^{2 k-3}}<$ $\delta<c \cdot 2^{-k}$.

Lemma 3 For $n \geq 12$, let $m=[n / 6]$, and

$$
E_{n}=\operatorname{cov}\left(D_{m}\right)+\operatorname{cov}\left(D_{m+1}\right)+\operatorname{cov}\left(D_{m+2}\right)+\cdots+\operatorname{cov}\left(D_{n}\right) .
$$

Then for some constant $c>0$,

$$
\log N\left(E_{n}, \sqrt{n} \cdot 2^{-2 n-1}\right) \geq c n \cdot 2^{4 n} .
$$

Proof: $\quad$ By Lemma 2, for each $m \leq k \leq n$, there exists a set $S_{k} \subset$ $\operatorname{cov}\left(D_{k}\right)$ of cardinality $L=e^{c \cdot 2^{4 n}}$ whose mutual distance between any two elements is at least $2^{-2 n}$. Consider the set

$$
F_{n}=S_{m}+S_{m+1}+\cdots+S_{n}
$$

For $t, s \in F_{n}$, suppose

$$
t=t_{m}+t_{m+1}+\cdots+t_{n}, \text { and } s=s_{m}+s_{m+1}+\cdots+s_{n}
$$


with $t_{k} \in S_{k}$ and $s_{k} \in S_{k}$. Define the Hamming distance

$$
h(t, s)=\text { cardinality of }\left\{k: t_{k} \neq s_{k}, m \leq k \leq n\right\} .
$$

For each $t \in F_{n}$, the ball

$$
B_{h}(t, n / 3):=\left\{s \in F_{n}: h(t, s) \leq n / 3\right\}
$$

contains no more than $(n L)^{n / 4}<L^{n / 3}$ elements. Thus $F_{n}$ contains a subset of cardinality $L^{n-m} \div L^{n / 3} \geq L^{n / 2}$, whose mutual Hamming distance between any two elements is at least $n / 4$. Thus the mutual $l_{2}$-distance is at least $\sqrt{n} \cdot 2^{-2 n-1}$. This implies that

$$
\log N\left(F_{n}, \sqrt{n} \cdot 2^{-2 n-1}\right) \geq \frac{n}{2} \log L=\frac{c n}{2} 2^{4 n} .
$$

Now we finish the proof of (ii). For any $0<\varepsilon<2^{-24}$, there exists $n \geq 12$, such that

$$
\sqrt{n+1} \cdot 2^{-2 n-3}<\varepsilon \leq \sqrt{n} \cdot 2^{-2 n-1}
$$

Because $F_{n} \subset \operatorname{cov}(T)$, we have

$$
\begin{aligned}
\log N(\operatorname{cov}(T), \varepsilon) & \geq \log N\left(F_{n}, \sqrt{n} \cdot 2^{-2 n-1}\right) \\
& \geq \frac{c n}{2} 2^{4 n} \\
& \geq c^{\prime} \varepsilon^{2}\left(\log \varepsilon^{-1}\right)^{-2}
\end{aligned}
$$

Acknowledgment The author thanks J. Creutzig, W. Li and W. Linde for there interest and valuable comments. 


\subsection{Reference}

[BLL] B. Bühler, W. Li and W. Linde, Location of Majorizing Measures, Asymptotic Methods in Probability and Statistics with Applications, Birkhauser, Boston.(to appear)

[BP] K. Ball and A. Pajor, The entropy of convex bodies with "few" extreme points, London Math. Soc. Lecture Note Ser. 158(1990), 25-32.

[C] B. Carl, Metric entropy of convex hulls in Hilbert spaces, Bull. London Math. Soc. 29(1997), 452-458.

[CKP] B. Carl, I. Kyrezi and A. Pajor, Metric entropy of convex hulls in Banach spaces, preprint, 1997.

[D] R. M. Dudley, The sizes of compact subsets of Hilbert space and continuity of Gaussian processes, J. Funct. Anal. 1(1967), 290-330.

[LL] W. Li and W. Linde, Metric Entropy of Convex Hulls in Hilbert Spaces, Studia Mathematica (to appear).

Fuchang Gao

Department of Mathematics

University of Idaho

Moscow, Idaho 83844-1103

fuchang@uidaho.edu 